\title{
Development of an Automatic Test System for the PMTs used in the BGO ECAL of DAMPE
}

\author{
Jianing Dong ${ }^{1}$ \\ Department of Physics, University of Science and Technology of China \\ State Key Laboratory of Particle Detection and Electronics(IHEP-USTC) \\ Hefei, 230026, China \\ E-mail: jndongemail.ustc.edu.cn
}

\section{Yunlong Zhang*}

Department of Modern Physics, University of Science and Technology of China State Key Laboratory of Particle Detection and Electronics(IHEP-USTC)

Hefei, 230026, China

E-mail: ylzhang@ustc.edu.cn

\section{Sicheng Wen}

Department of Modern Physics, University of Science and Technology of China State Key Laboratory of Particle Detection and Electronics(IHEP-USTC)

Hefei, 230026, China

E-mail: wenscheustc.edu.cn

\section{Zhiyong Zhang}

Department of Modern Physics, University of Science and Technology of China State Key Laboratory of Particle Detection and Electronics(IHEP-USTC)

Hefei, 230026, China

E-mail: zhzhyemail.ustc.edu.cn

\section{Zhongtao Shen}

Department of Modern Physics, University of Science and Technology of China State Key Laboratory of Particle Detection and Electronics(IHEP-USTC)

Hefei, 230026, China

E-mail: henzt@mail.ustc.edu.cn

\section{Changqing Feng}

Department of Modern Physics, University of Science and Technology of China State Key Laboratory of Particle Detection and Electronics(IHEP-USTC)

Hefei, 230026, China

E-mail: fengcqlustc.edu.cn

\section{Xiaolian Wang}

Department of Modern Physics, University of Science and Technology of China State Key Laboratory of Particle Detection and Electronics(IHEP-USTC)

\footnotetext{
${ }^{1}$ Speaker; *Corresponding Author
} 
Hefei, 230026, China

E-mail: wangxlaustc.edu.cn

\section{Zizong Xu}

Department of Modern Physics, University of Science and Technology of China State Key Laboratory of Particle Detection and Electronics(IHEP-USTC)

Hefei, 230026, China

E-mail: zzxu@ustc.edu.cn

\section{Shubin Liu}

Department of Modern Physics, University of Science and Technology of China

State Key Laboratory of Particle Detection and Electronics(IHEP-USTC)

Hefei, 230026, China

E-mail: liushboustc.edu.cn

An automatic system has been developed for the batch test of the photomultiplier tubes (PMTs) in the BGO electromagnetic calorimeter (ECAL) of the DArk Matter Particle Explorer (DAMPE). There are 616 PMTs (Hamamatsu R5610A-01) used in the BGO ECAL, which are critical for the realization of high dynamic readout and high precision measurement of the scintillation light from BGO crystals. In order to cover the large dynamic range of energy measurement of DAMPE, signals are read out from three dynodes of the PMTs. The charge ratios of the dynodes are of paramount importance to the energy reconstruction of high energy incident particles so that all the PMTs must be tested and calibrated. In addition, considering of the high reliability and quality requirements as a space-borne experiment, over 800 PMTs have been tested during the mass production and screening procedure both for the Qualification Model and the Flight Model. Therefore, a light-emitting diode (LED) based system has been designed to test the performance of PMTs automatically.

The test system is composed of a signal generator, a LED driver module, a dark box and a readout system which consists of a front end electronics (FEE) board, a data acquisition (DAQ) board and a data acquisition software based on Labwindows/CVI. An arbitrary waveform generator drives the LED source for illuminating 22 PMTs through optical fibers in one dark box. Then 66 dynode signals are read out by a FEE board, sent to DAQ module, stored in the computer and finally analyzed with a root program. As two dark boxes can be controlled simultaneously by the readout system, it takes about $\sim 30$ minutes to test 44 PMTs per time, which reduces the workload greatly and guarantees the project schedule. The details about this system and the test results are presented in this paper.

The 34th International Cosmic Ray Conference

30 July- 6 August, 2015

The Hague, The Netherlands 


\section{Introduction}

DAMPE is a detection satellite put forward by the Chinese Academy of Science which will be launched in 2015-2016. DAMPE aims to measure the dark matter indirectly through detecting high energy gamma-ray, electron and cosmic rays and it has the highest dynamic energy range from $5 \mathrm{GeV}$ to $10 \mathrm{TeV}$ in the world today [1][2]. As shown in Fig. 1, DAMPE is consist of plastic scintillator strips detector (PSD), silicon-tungsten tracker-converter (STK), BGO electromagnetic calorimeter (BGO ECAL) and neutron detector, in which BGO ECAL is the crucial sub-detector to realize the high dynamic energy range.

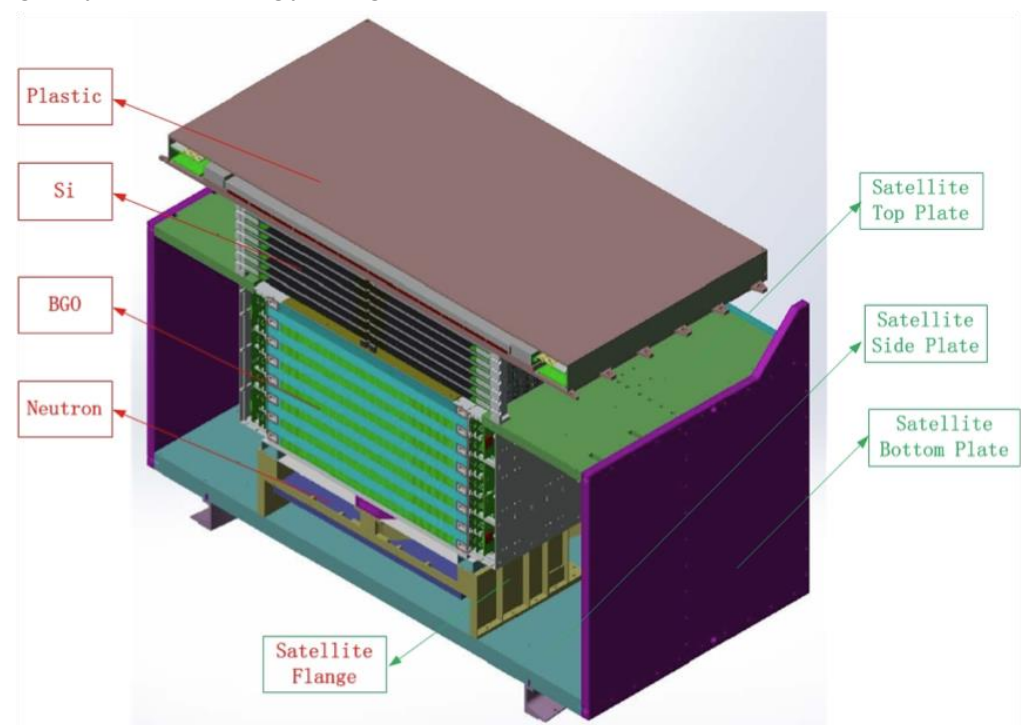

Fig. 1. The schematic view of DAMPE. It consists of PSD, STK, BGO ECAL and neutron detector.

The BGO ECAL is composed of 308 BGO bars and 616 PMTs to read out all the BGO signals. Then 800 PMTs have been tested during the mass production and screening procedure both for the Qualification Model and the Flight Model to insure the allowance. It spends a lot of time if the PMTs are tested one by one, so an automatically test system based on LED has been designed to test the performance of all the PMTs. And in this paper, the automatic test system and the test results are presented in detail.

\section{The BGO ECAL}

As shown in Fig. 2, the BGO ECAL is composed of 14 layers of BGO crystal and 22 BGO bars with a size of $2.5 \mathrm{~cm} * 2.5 \mathrm{~cm} * 60 \mathrm{~cm}$ for each bar are placed in each layer. Each BGO bar is viewed by two PMTs, from both sides respectively [3]. So 616 PMTs are required in the BGO ECAL. 


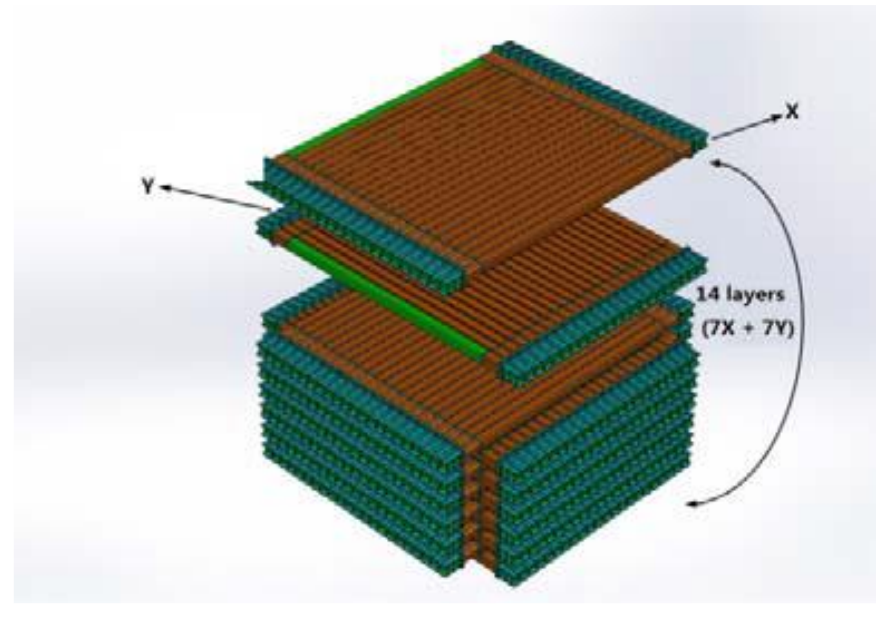

Fig. 2. Arrangement of 308 BGO crystal bars in the BGO ECAL.

According to the Monte Carlo simulation, the maximum deposited energy in one BGO bar is about $2 \mathrm{TeV}$ for a $10 \mathrm{TeV}$ high energy electron as the requirement of high dynamic energy range $(5 \mathrm{GeV}-10 \mathrm{TeV})$. The deposited energy from a minimum ionizing particle (MIP) passing through the $2.5 \mathrm{~cm}$ thick BGO bar is about $23 \mathrm{MeV}$. To precisely sample the cosmic ray shower profile, a lower energy limit less than $0.5 \mathrm{MIPs}$ is needed. So a measurement energy range from $11 \mathrm{MeV}$ to $2 \mathrm{TeV}$ is required for each $\mathrm{BGO}$ bar, which is equal to about $2 \times 10^{5}$ in dynamic range. Considering that the capability of one single electronics channel is about $10^{3}$ mostly, the relative gain of the PMT must be guaranteed to be greater than $10^{3}$.

The type of the PMTs used in BGO ECAL is R5610A-01, made by Hamamatsu [4], which has 10 stages of charge amplification. To achieve the relative gain of $10^{3}$, the three-dynode (Dy2, Dy5, Dy8) readout design of the PMT base board has been put forward [5]. Then the relative gain ratio of Dy8 to Dy5 and Dy5 to Dy2 can be tested and calibrated during the mass production to ensure the large dynamic range and the linearity of every PMT. Considering of the high reliability and quality requirements as a space-borne experiment, 800 PMTs have been tested and the proper 616 PMTs have been picked up and matched to their corresponding BGO bars. Then our object of high dynamic range measurement can be realized.

\section{The Automatic Test System}

As 800 PMTs must be tested both for the Qualification Model and the Flight Model, the mass measurement and time limitation in the engineer project are what we have to consider. So an automatic test system based on LED has been designed, which can measure 44 PMTs at a time and save a lot of time in the production.

The LED based test system is made up of a signal generator, a LED driver module, two dark boxes, and a readout system, as can been seen in Fig. 3. And in the dark box (Fig. 4), an integrating sphere with a LED in it has two bundles of optical fibers to put out the light uniformly, and 22 of them are connected to 22 grooves fixed in the dark box respectively, in which the PMT can be placed and be illuminated. Then all the 66 dynode signals can be read out by FEE board, sent to DAQ module, stored in the computer and finally analyzed with a root program. And 22 voltage signals are connected to the high voltage fan-out board to get the high voltage for the PMTs. 


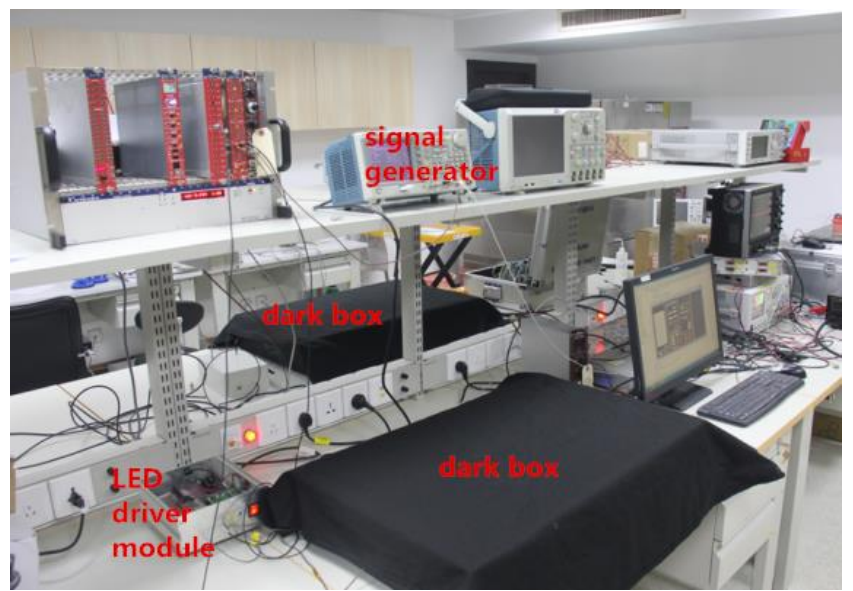

Fig. 3. Composition of the LED test system. The system is composed of a signal generator, LED driver module, two dark boxes and readout system.

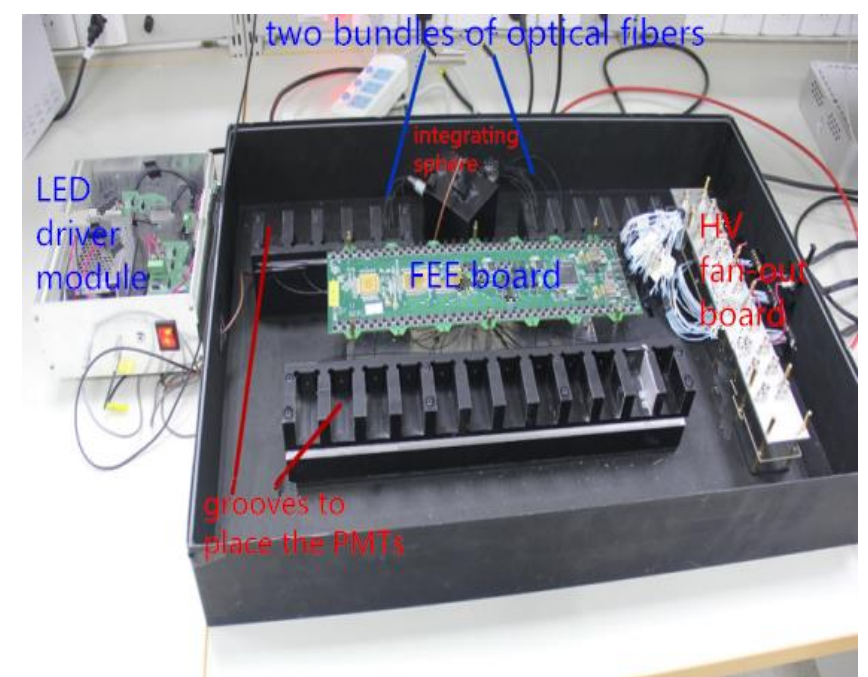

Fig. 4. Schematic view of the dark box. It consists of an integrating sphere, two bundles of optical fibers, 22 grooves to place the PMTs, a FEE board and a high voltage (HV) fan-out board.

In the automatic test system, as shown in Fig. 5, an arbitrary waveform generator (GEN) drives the LED source emit whose light shape is similar to the BGO fluorescence's through the LED driver module [6]. And 22 PMTs in the grooves can be illuminated by the optical fibers. The amplification of light can be increased through raising the generator output signal continuously, which can be controlled by the software based on Labwindows/CVI automatically. Then the ADC count of Dy8, Dy5, and Dy2 can be obtained from the readout system and the relative gain ratio of the PMT can be analyzed. As two dark boxes can be controlled simultaneously by the readout system, it takes about 30 minutes to test 44 PMTs per time, which reduces the workload greatly and guarantees the project schedule. 


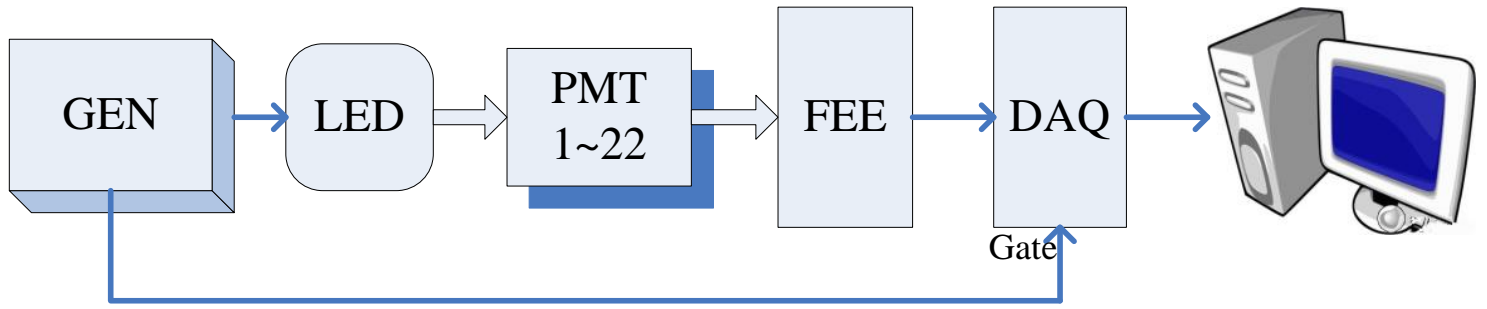

Fig. 5. Block diagram of the LED test system in one dark box. An arbitrary waveform generator (GEN) drives the LED emit under the driven of the LED driver module, so 22 PMTs can be illuminated through the optical fibers. Then 66 dynode signals can be read out by FEE board and DAQ board when the GEN gives a trigger to the DAQ board, and then the data of the dynode signals can be analyzed in the computer.

\section{Test Results}

All the PMTs' relative gain ratio of Dy8 to Dy5 and Dy5 to Dy2 have been tested in the automatic system, and the gain ratio of Dy8 to Dy5 and Dy5 to Dy2 can be calculated as:

$$
\text { Ratio }_{8-5}=\frac{A D C_{\text {count } 8}}{A D C_{\text {count } 5}} \text { and } \text { Ratio }_{5-2}=\frac{A D C_{\text {count } 5}}{A D C_{\text {count } 2}}
$$

Where $A D C_{\text {counts }}$ is the ADC count of Dy 8 after subtracting the pedestal, $A D C_{\text {count }}$ is the ADC count of Dy5 after subtracting the pedestal, $A D C_{\text {count } 2}$ is the ADC count of Dy2 after subtracting the pedestal. So the dynamic range (DR) of the PMT can be calculated as:

\section{$\mathrm{DR}=$ Ratio $_{8-5} \times$ Ratio $_{5-2}$}

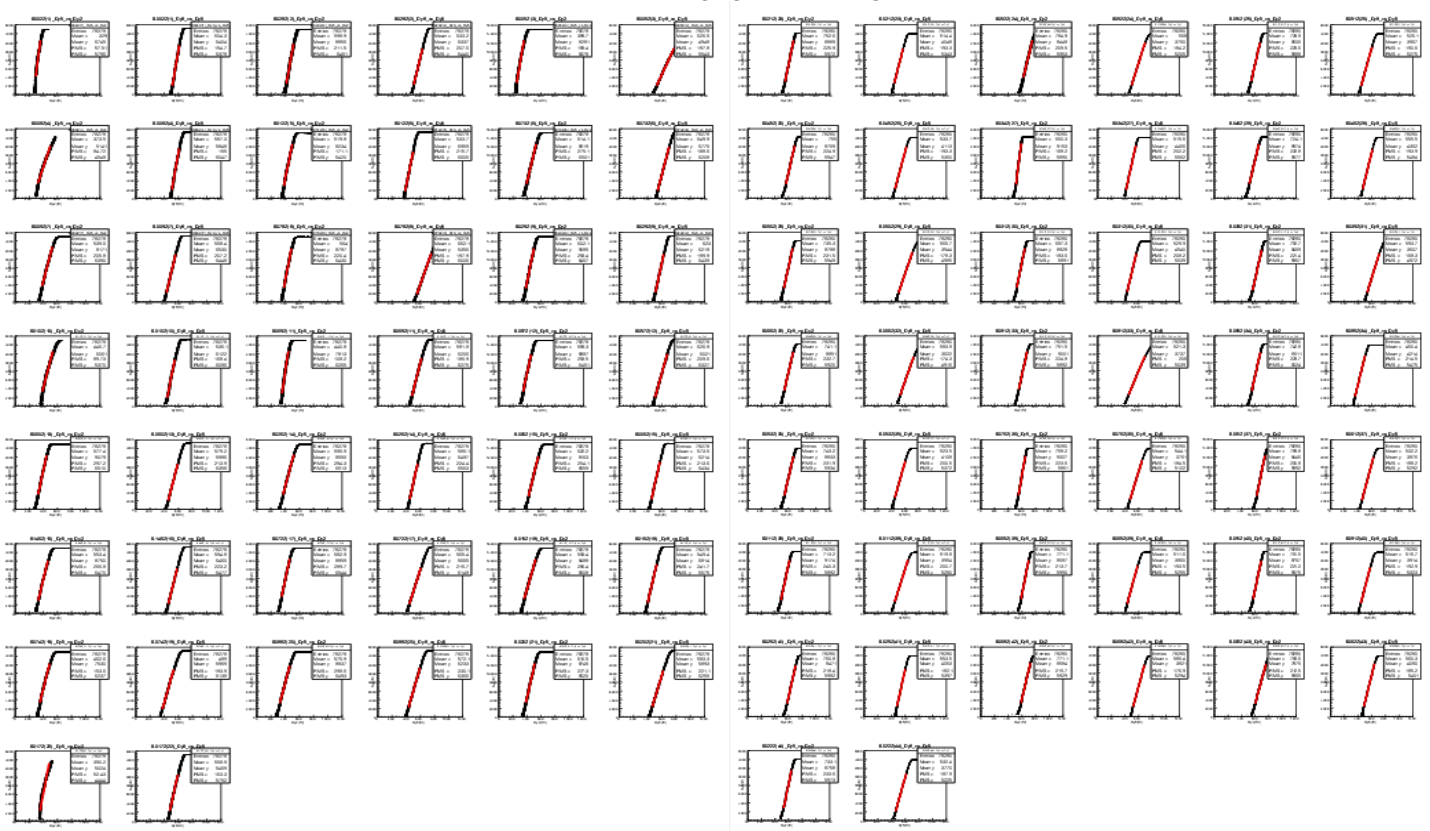

Fig. 6. The gain ratio of Dy8 to Dy5 and Dy5 to Dy2 of 44 PMTs which have been tested in the automatic LED system at a time. The dynamic ranges of all the PMTs are over 1000, and the linearities of most PMTs are well.

Fig.6 shows the gain ratios of 44 PMTs tested in the automatic system at a time under the high voltage of $670 \mathrm{~V}$ and the value of the gain ratio of Dy8 to Dy5 and Dy5 to Dy2 is commonly over 40, which makes the dynamic range of the PMT over 1000 and meets our need undoubtedly. Fig. 7 shows a typical PMT's gain ratio of Dy8 to Dy5 and Dy5 to Dy2, which should be linear. Otherwise, if a PMT does not perform well in its first test, it will be tested again and then be decided whether it is available or not. 

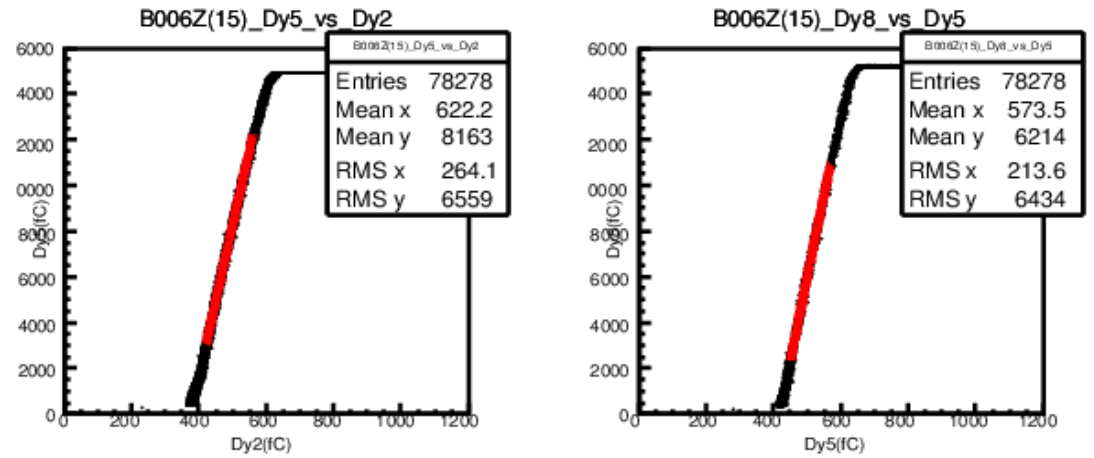

Fig. 7. The typical PMT's gain ratio of Dy8 to Dy5 and Dy5 to Dy2 whose linearity is well.

It can be seen from the figures that the relative gain ratio of the PMT can be easily over 1000, so almost all of the PMTs can pass this assessment and the parameters of the gain ratio can be saved and be prepared for the future experiment.

Therefore, it takes about 30 minutes to test 44 PMTs at a time and 19 times are needed to test all the 800 PMTs, which saves plenty of time for the project. And the PMTs perform well in the automatic LED system basically, which testify the feasibility of the automatic test system.

\section{Conclusion}

A LED based test system has been developed in this paper to measure the relative gain ratio of the PMT in bulk automatically. The performance of 800 PMTs have been measured in detail. And their test results have been stored in a database and will be used as the calibration values and analysis in future experiment. With the LED test system, 44PMTs can be tested at a time automatically, which saves a lot of time, reduces the workload greatly and guarantees the project schedule.

\section{Acknowledgement}

This work was supported by the Strategic Priority Research Program on Space Science of Chinese Academy of Sciences (Grant No. XDA04040202-4), and the National Basic Research Program (973 Program) of China (Grant No. 2010CB833002).

\section{References}

[1] Chang, J., et al. An excess of cosmic ray electrons at energies of 300-800 GeV, Nature 456.7220 (2008): 362-365.

[2] J. Chang, Dark Matter Particles Detection in Space, Journal of Engineering Studies. vol.2(2), pp. 95-99, June 2010.

[3] Zhang, Yun-Long, et al. A high dynamic range readout unit for a calorimeter, Chinese Physics C (HEP \& NP) 36.1 (2012): 71-73.

[4] http://www.hamamatsu.com/jp/en/product/category/3100/3001/R5610A/index.html

[5] Z. Zhang, Y. Zhang, et al. Design of a high dynamic range photomultiplier base board for the BGO ECAL of DAMPE, Nuclear Instruments and Methods in Physics Research A 780(2015) 21-26.

[6] T. Xiang, X. Jin, et al. Study of Linearity for a High Dynamic Range Calorimeter, Chinese Physics C 38.4(2014) 046201. 